\title{
Incidência de traumas faciais em atletas profissionais: revisão integrativa
}

\author{
Incidence of facial trauma in professional athletes: integrative review \\ Incidencia del trauma facial en atletas profesionales: revisión integradora
}

Recebido: 16/11/2021 | Revisado: 24/11/2021 | Aceito: 25/11/2021 | Publicado: 07/12/2021

\author{
Ernandi Ribeiro Cezar Lima \\ ORCID: https://orcid.org/0000-0002-6783-3627 \\ Centro Universitário Tiradentes, Brasil \\ E-mail: ernandicezar@outlook.com \\ Estéfane Carvalho do Nascimento \\ ORCID: https://orcid.org/0000-0002-4372-9657 \\ Centro Universitário Tiradentes, Brasil \\ E-mail: estefane.carvalho@outlook.com \\ Gabriela de Almeida Sousa \\ ORCID: https://orcid.org/0000-0001-8734-4831 \\ Universidade Federal de Alagoas, Brasil \\ E-mail:ggabrielalmeida1@gmail.com \\ Nathalia Santos \\ ORCID: https://orcid.org/0000-0001-5252-5894 \\ Centro Universitário Tiradentes, Brasil \\ E-mail: nathalia.rdi@gmail.com \\ Luciano Schwartz Lessa Filho \\ ORCID: https://orcid.org/0000-0001-6077-9333 \\ Centro Universitário Tiradentes, Brasil \\ E-mail: lucbmf@gmail.com
}

\begin{abstract}
Resumo
A incidência de traumas faciais vem crescendo ao redor do mundo por diversos fatores, dentre eles a prática esportiva. Lesões faciais podem ter consequências catastróficas para os atletas, sendo assim, é de extrema importância examinar as taxas, os fatores de risco e a natureza desses traumas. O objetivo deste presente estudo foi realizar uma investigação da incidência de traumas faciais, tais como as regiões anatômicas mais acometidas e tratamento através de uma revisão integrativa da literatura. Foi realizada uma revisão integrativa, por meio de pesquisas nas bases de dados SCIELO, PUBMED e MEDLINE, utilizando o operador boleano AND, sendo critérios de inclusão, artigos em inglês que falem sobre a incidência de traumas faciais em atletas, de categoria profissional, bem como as regiões anatômicas envolvidas, o esporte e o uso ou não de proteção facial e bucal. Á realização dos levantamentos bibliográficos ocorreu no mês de março de 2020. Analisaram-se 12 artigos que atenderam os critérios de inclusão. Os artigos revisados mostraram que o esporte que mais ocorre traumas faciais é o futebol, a região anatômica mais acometida foi o complexo zigomático, e a não utilização de medidas preventivas foi o principal fator para ocorrência dos traumas. Esta revisão revelou uma alta prevalência de lesões maxilofaciais, com prevalência maior na região da mandíbula e complexo zigomático.
\end{abstract}

Palavras-chave: Traumatismos faciais; Esportes; Traumatismos em atletas.

\begin{abstract}
The incidence of facial trauma has been growing around the world due to several factors, including sports practice. Facial injuries can have catastrophic consequences for athletes, so it is extremely important to examine the rates, risk factors and nature of these traumas. The aim of this study was to investigate the incidence of facial trauma, such as the most affected anatomical regions and treatment through an integrative literature review. An integrative review was carried out, through research in the SCIELO, PUBMED and MEDLINE databases, using the Bolean operator AND, and inclusion criteria were articles in English that talk about the incidence of facial trauma in athletes, of professional category, as well as the anatomical regions involved, the sport and the use or not of facial and oral protection. The bibliographic surveys took place in March 2020. We analyzed 12 articles that met the inclusion criteria. The reviewed articles showed that the sport that most occurs facial traumas is soccer, the most affected anatomical region was the zygomatic complex, and the non-use of preventive measures was the main factor for the occurrence of traumas. This review revealed a high prevalence of maxillofacial lesions, with higher prevalence in the mandible region and zygomatic complex.
\end{abstract}

Keywords: Facial injuries; Sports; Athletic injuries. 


\begin{abstract}
Resumen
La incidencia de traumatismos faciales está aumentando en todo el mundo debido a varios factores, entre ellos la práctica deportiva. Las lesiones faciales pueden tener consecuencias catastróficas para los atletas, por lo que es de vital importancia examinar las tasas, los factores de riesgo y la naturaleza de estas lesiones. El objetivo de este estudio fue realizar una investigación de la incidencia de traumatismos faciales, como las regions anatómicas más afectadas y su tratamiento através de una revisión integradora de la literatura. Se realizó una revisión integradora, mediante búsquedas en las bases de datos SciELO, PubMed y MEDLINE, utilizando el operador booleano "AND", como criterio de inclusión, artículos en inglés que hablan sobre la incidencia de trauma facial en deportistas, de categoría profesional, así como las regiones anatómicas involucradas, el deporte y el uso o no de protección facial y bucal. Las encuestas bibliográficas se realizaron en marzo de 2020. Se analizaron doce artículos que cumplieron con los criterios de inclusión. Los artículos revisados mostraron que el deporte con más traumatismos faciales es el fútbol, la región anatómica más afectada fue el complejo cigomático y la no utilización de medidas preventivas fue el principal factor para la ocurrencia de traumas. Esta revisión reveló una alta prevalencia de lesiones maxilofaciales, con una mayor prevalencia en la región mandibular y el complejo cigomático.
\end{abstract}

Palabras clave: Traumatismos faciales; Deportes; Traumatismos en atletas.

\title{
1. Introdução
}

O esporte no mundo todo está sendo cada vez mais praticado, e consequentemente ocasionando um maior índice de lesões associadas a prática esportiva também. Os esportes de contato mantiveram um lugar relevante na causa de trauma facial, fato que pode ser justificado, por ser uma área mais vulnerável e menos protegida do corpo (Tanaka, et al., 1996; Hill, et al., 1998; Ranali \& Demas 2002).

De acordo com diferentes estudos de setenta países, a incidência de traumas faciais sofridas durante o esporte são de 5,6 a 33\%, e com relação ao esporte varia da popularidade nos países (Lim, et al., 1993; Carroll, et al., 1995; Tanaka, et al., 1996; Maladiere, et al., 2001; Motamedi 2003; Delillbassi, et al., 2004). Dentre os esportes de contato, o futebol é o mais frequentemente praticado no Brasil (Santos, et al., 2004).

Frontera (2008) reuniu alguns fatores de risco que certamente estão relacionados com o traumatismo de modo geral e com o trauma esportivo; tais como: faixa etária entre 15 a 24 anos; posicionamento inadequado dos dentes na arcada; atletas que são respiradores bucais; nível de profissionalismo da competição; jogadores avançados nos esportes coletivos; uso de aparelho ortodôntico; e principalmente, o não uso dos equipamentos de proteção.

Embora algumas lesões faciais por práticas esportivas sejam inevitáveis, a maioria é evitável com a utilização adequada de protetores bucais e faciais. Entretanto, a maioria dos jogadores consideram o uso do protetor bucal desnecessário. Devido à falta de informação e de conhecimento, o uso de dispositivos de proteção não abrange a maioria dos atletas (Yamada, et al., 2008).

O objetivo deste presente estudo foi realizar uma investigação da incidência de traumas faciais, tais como os sítios mais acometidos e tratamento através de uma revisão integrativa da literatura.

\section{Metodologia}

Para realizar o levantamento bibliográfico foi utilizada as bases de dados National Library of Medicine (PubMed), Medical Literature Analysis and Retrieval System Online (MEDLINE) e Scientific Eletronic Liberay Online (Scielo). Na pesquisa foram inclusos somente artigos em idioma inglês publicados entre os anos de 1985 a 2019 , que abordaram a incidência de traumas faciais em atletas, de categoria profissional, bem como as regiões anatômicas envolvidas, o esporte e o uso ou não de proteção facial e bucal.

A estratégia de busca (Figura 1) foi realizada e adaptada com base nos Descritores em Ciências da Saúde - DeCS: Facial Injuries; Athletic Injuries; Sports. Para padronização das buscas nas bases de dados, foram utilizadas a seguinte combinação: "facial injuries" AND “athletic injuries" AND "sports"; "facial injuries". 
Ao inserir os descritores nas bases de dados, foram encontrados 1.053 artigos. A seleção foi realizada em etapas, inicialmente através da leitura do título e resumo do artigo, seguindo os critérios de inclusão, sendo excluídos estudos do tipo revisão de literatura, resumos, dissertações e teses, bem como os que não estavam de acordo com o tema proposto, obtendo um total de 49 artigos.

Após isso, foram excluídos os duplicados, o qual restaram 42 e por fim, removidos aqueles com falta de detalhes nos estudos epidemiológicos e mantendo os bem definidos, que totalizaram em 11 artigos.

Figura 1 - Esquematização da busca metodológica nas bases de dados.

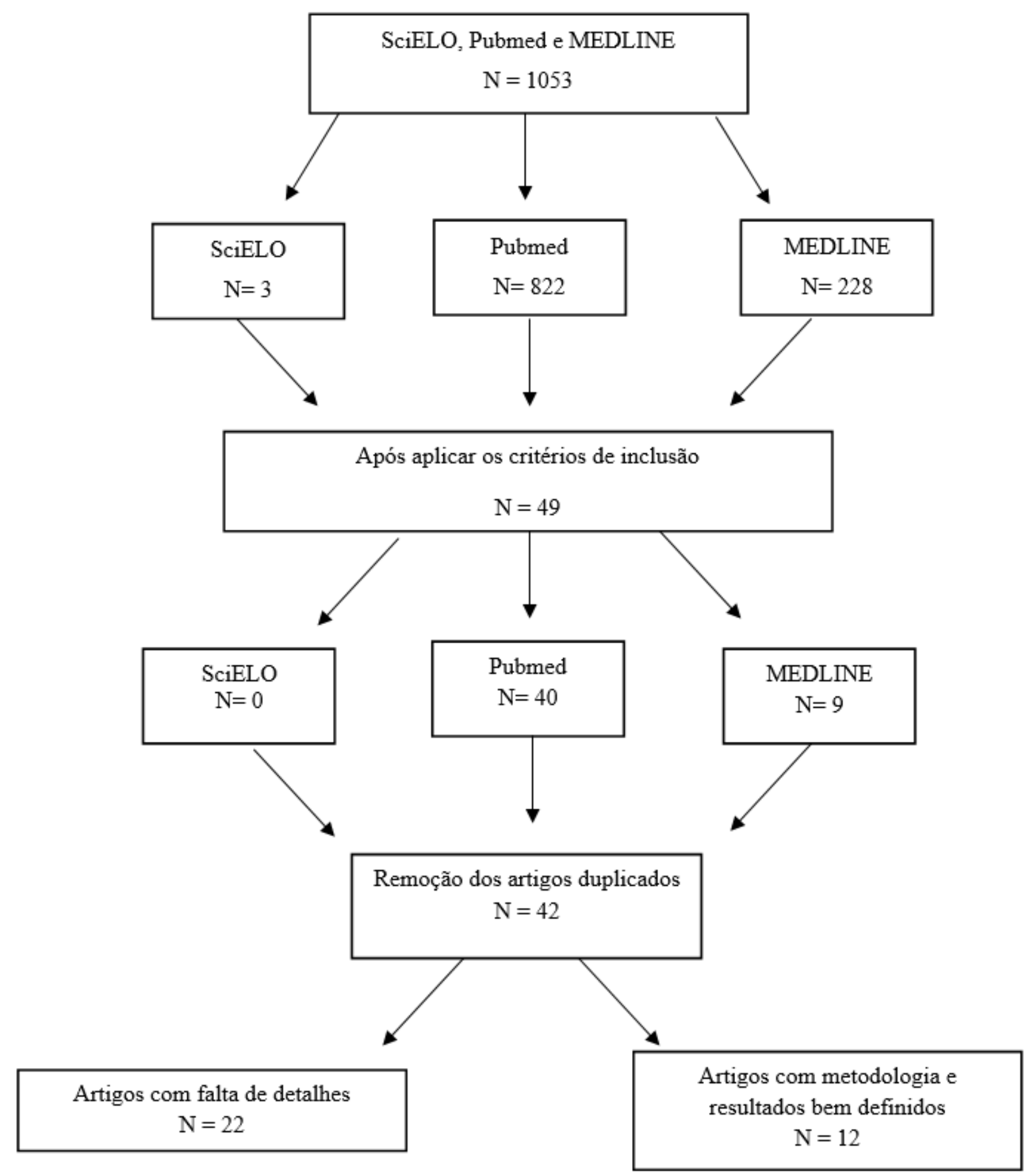

Fonte: Dados da pesquisa (2021). 


\section{Resultados}

Foram obtidos 12 artigos como produto da pesquisa efetuada aos critérios de inclusão estabelecidos anteriormente, além de passarem pelo processo de exclusão para assim atenderem os princípios de seleção referentes ao tema escolhido. Os dados da Tabela 1 apresentam características sucintas e centrais dos artigos analisados.

Tabela 1 - Resumo das informações dos artigos incluídos na Revisão Integrativa.

\begin{tabular}{|c|c|c|c|c|}
\hline TÍTULO & AUTOR & ANO & DELINEAMENTO/MÉTODOS & DESFECHO/RESULTADOS \\
\hline $\begin{array}{l}\text { Sports-Related } \\
\text { Maxillofacial } \\
\text { Fractures Over } \\
\text { an 11-Year } \\
\text { Period. }\end{array}$ & $\begin{array}{l}\text { Antoun, S.J; } \\
\text { Lee, K.H. }\end{array}$ & $\begin{array}{c}\text { 2008/New } \\
\text { Zealand }\end{array}$ & $\begin{array}{l}\text { A amostra retros-pectiva do estudo incluiu todos } \\
\text { os pacientes com fraturas faciais relacionadas ao } \\
\text { esporte atendidos na Unidade Oral e } \\
\text { Maxilofacial, do Hospital da Igreja, por um } \\
\text { período de } 10 \text { anos (1996 a 2006). De um total de } \\
2.582 \text { dos pacientes com fraturas maxilofaciais } \\
\text { identificadas do banco de dados, } 561 \text { eram } \\
\text { relacionados ao esporte }(21,7 \%) \text {. Os registros } \\
\text { incluíam dados sóciodemográficos básicos, } \\
\text { detalhes específicos de uma lesão, causa da lesão } \\
\text { esportiva, local da fratura e método de } \\
\text { tratamento. Pacientes com lesões de tecidos } \\
\text { moles não foram incluídos no estudo. }\end{array}$ & $\begin{array}{l}\text { Referente ao tipo de esporte como causa das } \\
\text { fraturas faciais, o principal foi o rugby }(52 \%) \text {, } \\
\text { seguido do ciclismo }(15,3 \%) \text {, críquete }(7,1 \%) \text { e } \\
\text { futebol }(4,8 \%) \text {. O local de fratura, a região de } \\
\text { terço médio foi responsável por } 56,8 \% \text { de todas } \\
\text { as estruturas, seguidos de fraturas mandibulares } \\
(41,4 \%) \text {, zigomático }(29,4 \%) \text { e orbital }(16,9 \%) \text {. } \\
\text { Com relação ao método de tratamento, o mais } \\
\text { utilizado foi a redução aberta }(60,3 \%) \text { e fixação } \\
\text { interna }(23,8 \%) \text {. }\end{array}$ \\
\hline $\begin{array}{l}\text { A survey of } \\
\text { orofacial } \\
\text { injuries among } \\
\text { basketball } \\
\text { players }\end{array}$ & Azodo et al. & $2011 /$ EUA & $\begin{array}{l}\text { A pesquisa foi realizada através de um } \\
\text { questionário sobre a prevalência de lesões } \\
\text { orofaciais e tipo de lesão, conscientização dos } \\
\text { atletas, atitudes e uso de protetores bucais. }\end{array}$ & $\begin{array}{l}\text { A prevalência de lesões faciais e orais entre os } \\
\text { entrevistados foi de } 62,8 \% \text { sendo o lábio e a } \\
\text { gengiva os locais mais comuns, respectivamente, } \\
\text { seguidos de fraturas em região Mandibular e } \\
\text { Zigomático. Mais da metade tinha conhecimento } \\
\text { sobre os protetores bucais e o treinador era a } \\
\text { principal fonte de informações. Os motivos da } \\
\text { não utilização dos protetores bucais eram } \\
\text { principalmente ignorância, indisponibilidade e } \\
\text { não acessibilidade. }\end{array}$ \\
\hline $\begin{array}{l}\text { Maxillofacial } \\
\text { fractures } \\
\text { sustained during } \\
\text { sports played } \\
\text { with a ball }\end{array}$ & $\begin{array}{c}\text { Delilbasi et } \\
\text { al. }\end{array}$ & 2004/USA & $\begin{array}{l}\text { A pesquisa consistiu em avaliar arquivos de } \\
\text { pacientes que sofreram fraturas durante } \\
\text { atividades esportivas que deram entrada no } \\
\text { Departamento de Cirurgia Oral e Maxilo facial, } \\
\text { Faculdade Odontologia da Universidade de } \\
\text { Osaka, durante o período de } 1986 \text { a 2002. Os } \\
\text { arquivos foram avaliados considerando idade e } \\
\text { sexo, local da fratura, o mês e o ano em que a } \\
\text { lesão ocorreu e a modalidade de tratamento. } \\
\text { Neste estudo, apenas fraturas maxilofaciais } \\
\text { foram investigadas; consequentemente, lesões de } \\
\text { tecidos moles (abrasão e laceração) não foram } \\
\text { incluídas. }\end{array}$ & $\begin{array}{l}\text { As fraturas principais resultaram do beisebol } \\
(44 \%) \text {, seguido pelo rugby }(28 \%) \text {, futebol }(18 \%) \\
\text { e outros esportes relacionados à bola, como } \\
\text { basquete, golfe, tênis e handebol ( } 10 \%) \text {. A } \\
\text { maioria dos pacientes apresentava fratura } \\
\text { mandibular (56\%), seguida por fraturas de terço } \\
\text { médio ( } 31 \%) \text {. Dos pacientes que necessitaram de } \\
\text { intervenção cirúrgica para tratamento das fraturas } \\
\text { equivaleu a 58,9\%. A causa mais comum das } \\
\text { fraturas foi o impacto contra outro jogador }(43 \%) \\
\text { seguido de impacto contra o equipamento }(37 \%) \text {. }\end{array}$ \\
\hline $\begin{array}{l}\text { Maxillofacial } \\
\text { and dental } \\
\text { soccer injuries } \\
\text { in finland }\end{array}$ & $\begin{array}{c}\text { Sane, J. ; } \\
\text { Ylippavalnie } \\
\text { m, P. }\end{array}$ & $\begin{array}{c}\text { 1987/Finlan } \\
\text { d }\end{array}$ & $\begin{array}{l}\text { A metodologia consistiu em analisar registros } \\
\text { médicos de todos os jogadores de futebol da } \\
\text { Finlândia durante os anos 1979-1982. Houve } \\
8640 \text { acidentes durante partidas ou treinamentos. } \\
552 \text { acidentes }(6,4 \%) \text { afetaram as regiões } \\
\text { maxilofaciais. Foram localiza-dos registros } \\
\text { médicos referentes a } 537 \text { casos }(97 \%) \text { e foram } \\
\text { utilizados neste estudo. A idade média dos } \\
\text { jogadores lesiona-dos foi de } 25 \text { anos. Apenas } \\
0,6 \% \text { dos jogadores eram do sexo feminino. }\end{array}$ & $\begin{array}{l}\text { Entre os } 537 \text { jogadores que sofreram lesões } \\
\text { maxilofaciais e dentárias, houve } 843 \text { lesões } \\
\text { separadas dos tecidos duros maxilofaciais e } \\
\text { dentários e próteses dentárias. Abrasões e } \\
\text { lacerações foram excluídas. } 681 \text { dos ferimentos } \\
(80,8 \%) \text { afetaram os dentes ou processos } \\
\text { alveolares e } 95(11,2 \%) \text { eram fraturas do terço } \\
\text { inferior ou médio. O tratamento foi conservador } \\
\text { em } 40,2 \% \text { dos participantes. }\end{array}$ \\
\hline $\begin{array}{l}\text { Maxillofacial } \\
\text { Fractures } \\
\text { Sustained } \\
\text { During Sports }\end{array}$ & Tanaka et al., & 1996/Japan & $\begin{array}{l}\text { A pesquisa consistiu em avaliar entre o período } \\
\text { de } 1977 \text { e 1993, } 98 \text { casos com fraturas } \\
\text { Maxilofaciais ocasionadas durante atividade } \\
\text { esportiva, tratadas no Departamento de Cirurgia } \\
\text { Maxilofacial, Faculdade de Odontologia em } \\
\text { Tóquio. Os fatores considerados para análise } \\
\text { foram o tipo de esporte envolvido, idade e sexo, } \\
\text { causa do acidente, local da lesão, e modo de } \\
\text { tratamento. }\end{array}$ & $\begin{array}{l}\text { A incidência de fratura era mais comum no rugby } \\
\text { e esqui, seguido de beisebol e futebol. A maioria } \\
\text { dos pacientes apresentava fratura mandibular } \\
(60,2 \%) \text {, seguidos por fraturas alveolares } \\
(22,4 \%) \text {. O tratamento conservador foi realizado } \\
\text { na maioria dos casos. A causa mais comum foi o } \\
\text { impacto contra outro jogador }(56,1 \%) \text {. }\end{array}$ \\
\hline $\begin{array}{l}\text { Dental and } \\
\text { facial injuries } \\
\text { following sports } \\
\text { accidents: a } \\
\text { study of } 130 \\
\text { patients }\end{array}$ & $\begin{array}{l}\text { Hill, CM; } \\
\text { Crosher, R.F; } \\
\text { Mason, } \\
\text { D.A }\end{array}$ & $\begin{array}{c}\text { 1985/Franc } \\
\mathrm{e}\end{array}$ & $\begin{array}{l}\text { Dados de pacientes que sofreram lesões por } \\
\text { esportes foram coletados em cartões de registro } \\
\text { projetados. As informações incluíam idade, sexo, } \\
\text { estado civil, causa, natureza e tratamento de } \\
\text { lesões, juntamente com informações hospitalares } \\
\text { e radiográficas. Para ser incluído neste estudo os } \\
\text { pacientes devem ter sofrido uma lesão nos } \\
\text { tecidos duros (isto é, dentes e } \\
\text { esqueleto), excluindo abrasões e lacerações. }\end{array}$ & $\begin{array}{l}\text { As mais comuns foram as fraturas do malar } \\
\text { (zigoma) observado } 41 \% \text { dos pacientes, seguida } \\
\text { de fraturas da mandíbula em } 31 \% \text { e fraturas } \\
\text { dentárias e alveolares em } 26 \% \text { dos casos. Os três } \\
\text { esportes coletivos mais praticados na França } \\
\text { (rugby, críquete e futebol) foram o que mais } \\
\text { apresentou as lesões. } 51 \% \text { dos casos exigiam } \\
\text { medidas conservadoras como tratamento, e } 37 \% \\
\text { tratados cirurgicamente. }\end{array}$ \\
\hline
\end{tabular}




\begin{tabular}{|c|c|c|c|c|}
\hline $\begin{array}{l}\text { Facial Injuries } \\
\text { in Handball: A } \\
\text { Survey of } \\
\text { Handball } \\
\text { Coaches }\end{array}$ & $\begin{array}{c}\text { Hwang, K; } \\
\text { Kim, H. }\end{array}$ & 2019 & $\begin{array}{l}\text { Foram impressos questionários e entregues a } 45 \\
\text { treinadores de handebol, o qual nesse } \\
\text { questionário continha perguntas sobre lesões } \\
\text { faciais que seus jogadores já haviam tido, } \\
\text { posição do jogador, etiologia, local e tipo de } \\
\text { lesão. }\end{array}$ & $\begin{array}{l}\text { O nariz foi o local mais comum das lesões } \\
(57,7 \%) \text {, seguido de órbita }(19,6 \%) \text { e zigomático } \\
(12,5 \%) \text {. A posição do jogador, } 21,4 \% \text { das lesões } \\
\text { foram em Zagueiros. Com relação ao uso de } \\
\text { proteção bucal e facial, nenhum jogador utilizava. } \\
\text { A causa mais comum foram por mão e cotovelo, } \\
26,8 \% \text { e } 12,5 \% \text { respectivamente. }\end{array}$ \\
\hline $\begin{array}{l}\text { Etiology and } \\
\text { incidence of } \\
\text { facial fractures } \\
\text { sustained during } \\
\text { sports: a } \\
\text { prospective } \\
\text { study of } 140 \\
\text { patients }\end{array}$ & $\begin{array}{l}\text { Maladiere et } \\
\text { al., }\end{array}$ & $\begin{array}{l}\text { 2001/ } \\
\text { France }\end{array}$ & $\begin{array}{l}\text { Um estudo prospectivo foi utilizado para todos } \\
\text { pacientes admitidos no Departamento de Cirurgia } \\
\text { Oral e Maxilofacial. Os registros foram avaliados } \\
\text { para os seguintes dados: idade, sexo, local da } \\
\text { fratura e causa da lesão (tipo de esporte e } \\
\text { circunstâncias). }\end{array}$ & $\begin{array}{l}\text { A fratura mandibular foi a mais comum com } \\
34,4 \% \text {, seguidos de fraturas do osso zigomático } \\
\text { em } 23,4 \% \text {, nasal em } 15,6 \% \text { e arco zigomático em } \\
6,5 \% \text { dos casos. A incidência de fratura foi mais } \\
\text { comum no futebol } 25 \% \text {, seguido do rugby } 15 \%\end{array}$ \\
\hline $\begin{array}{l}\text { Sports-related } \\
\text { maxillofacial } \\
\text { fractures: A } \\
\text { retrospective } \\
\text { study of } 125 \\
\text { patients }\end{array}$ & $\begin{array}{l}\text { Mourouzis, } \\
\text { C. Koumoura, } \\
\text { F. }\end{array}$ & $\begin{array}{l}2005 \text { / } \\
\text { Greece }\end{array}$ & $\begin{array}{l}\text { Durante o período } 1996-2002,125 \text { pacientes que } \\
\text { sofreram fraturas faciais como resultado de } \\
\text { atividades atléticas foram tratados em no } \\
\text { departamento dos autores. Os fatores } \\
\text { considerados para as análises foram idade, sexo, } \\
\text { tipo de esporte envolvido, mecanismo de lesão, } \\
\text { local de fraturas, e tratamento. }\end{array}$ & $\begin{array}{l}\text { O futebol foi o esporte mais comum relacionado a } \\
\text { fraturas faciais em } 64 \% \text { dos casos, seguido de } \\
\text { basquete } 13,6 \% \text {, taekwondo } 4,8 \% \text { e esqui } 3,2 \% \text {. } \\
\text { A maioria das fraturas foram em região } \\
\text { mandibular } 45,94 \% \text {, seguida por zigoma. O } \\
\text { tratamento mais utilizado em } 60 \% \text { foi cirúrgico, e } \\
40 \% \text { conservador. }\end{array}$ \\
\hline $\begin{array}{c}\text { Sports-Related } \\
\text { Maxillofacial } \\
\text { Injuries }\end{array}$ & Murphy et al., & $\begin{array}{c}2015 \text { / } \\
\text { Irlanda }\end{array}$ & $\begin{array}{l}\text { Foi realizada uma revisão retrospectiva de todas } \\
\text { as lesões maxilo-faciais em um serviço de } \\
\text { trauma. Os registros foram revisados e as } \\
\text { seguintes variáveis foram registradas: idade, } \\
\text { sexo, esporte envolvido, lesão sofrida, } \\
\text { mecanismo de lesão, tratamento, participação } \\
\text { subsequente e intervalo antes do retorno ao } \\
\text { esporte }\end{array}$ & $\begin{array}{l}\text { As lesões esportivas mais comuns foram as } \\
\text { seguintes: futebol gaélico } 35,3 \% \text {, futebol } 22,3 \% \text {, } \\
\text { rugby } 12,4 \% \text { e esportes equinos } 12,4 \% \text {. A lesão } \\
\text { mais comum sofrida foi a fratura do complexo } \\
\text { zigomático } 36,4 \% \text {. Ocorreu fratura mandibular } \\
\text { em } 20 \% \text {, fratura orbital em } 14,2 \% \text { e fratura nasal } \\
\text { em } 12,3 \% \text {. }\end{array}$ \\
\hline $\begin{array}{l}\text { Sport-Related } \\
\text { Maxillofacial } \\
\text { Fractures }\end{array}$ & Ruslin et al., & $\begin{array}{c}2016 \text { / } \\
\text { Holanda }\end{array}$ & $\begin{array}{l}\text { Este estudo baseia-se na análise de um banco de } \\
\text { dados de pacientes do Departamento de Cirurgia } \\
\text { Oral e Maxilofacial da Vrije University Medical } \\
\text { Center (VUmc), os dados coletados foram: sexo, } \\
\text { idade, tipo de esporte e tipo de fratura } \\
\text { maxilofacial. }\end{array}$ & $\begin{array}{l}\text { O futebol foi a principal causa do trauma } \\
\text { maxilofacial }(28 \%) \text {, seguido do hóquei em campo } \\
(25 \%) \text {. As fraturas mais comumente observadas } \\
\text { foram relacionadas ao complexo zigomático } \\
(45 \%) \text {, seguidos por fraturas mandibulares }(32 \%) \text {. }\end{array}$ \\
\hline $\begin{array}{l}\text { The incidence } \\
\text { of } \\
\text { head/neck/orofa } \\
\text { cial injuries in } \\
\text { non-elite } \\
\text { Australian } \\
\text { Football }\end{array}$ & $\begin{array}{l}\text { Braham, R.; } \\
\text { Finch, CF; } \\
\text { McCrory, P }\end{array}$ & $\begin{array}{c}2004 / \\
\text { Australian }\end{array}$ & $\begin{array}{l}\text { Tratou-se de um estudo controlado projetado } \\
\text { para avaliar a incidência e fatores de risco e a } \\
\text { eficácia dos protetores bucais para prevenir } \\
\text { lesões no futebol australiano. Essas lesões } \\
\text { incluíam qualquer lesão em região frontal, couro } \\
\text { cabeludo, orelhas, crânio, cérebro e face. As } \\
\text { taxas de lesões foram calculadas como o número } \\
\text { de lesões por } 1.000 \text { jogadores. }\end{array}$ & $\begin{array}{l}\text { Dos jogadores que participaram do estudo, } 294 \\
\text { não usavam proteção facial ou bucal em nenhum } \\
\text { momento. O diagnóstico mais comum foi } \\
\text { laceração facial seguido de concussão. Todos os } \\
\text { jogadores que sofreram concussão fizeram isso } \\
\text { durante a competição. } 9(24,3 \%) \text { jogadores com } \\
\text { lesões foram encaminhados para o hospital para } \\
\text { tratamento adicional. } 4 \text { tiveram cortes, lacerações } \\
\text { e um apresentava fratura. }\end{array}$ \\
\hline
\end{tabular}

Fonte: Dados da pesquisa (2021).

\section{Discussão}

De todas as fraturas maxilofaciais tratadas e estudadas, cerca de $20 \%$ estavam relacionadas ao esporte e entre eles, o esporte mais comum era o futebol.

A causa mais comum dessas fraturas, são descritas por Tanaka, et al., (1996), Delilbasi, et al., (2004) e Hwang e Kim, (2019), os quais descrevem que o impacto contra outro jogador ou contra o equipamento são as principais etiologias dos comprometimentos maxilofaciais de caráter grave ou não grave, pois, de acordo com a fricção entre dois corpos que estão em desarmonia, uma resistência oposta ao movimento é realizada e como resultado, há uma fratura.

Os locais mais comumente afetados diante dos traumas faciais são o complexo zigomático e as fraturas mandibulares. Murphy, et al., (2015), Ruslin, et al., (2016), Hill, et al., (1985), Mourouzis e Koumoura, (2005) e Maladiere, et al., (2001) concordam com essa afirmação quando destacam que em suas pesquisas, em média $45 \%$ dos envolvidos apresentavam essas estruturas comprometidas de forma isolada ou múltipla.

Azodo et al., (2011), relata ainda, em um estudo realizado com jogadores de basquete, que as taxas de lesões foram maiores em profissionais, quando comparadas aos jogadores não profissionais, mas, a minoria desses jogadores foram 
hospitalizados, ou seja, a maior parte não apresentavam lesões graves. Outro estudo feito por Hwang e Kim, (2019), também demonstra a prevalência desses traumas no handebol e observa-se que a ocorrência destes, também era menor ou de caráter não grave.

Dessa forma, e de acordo com os resultados supracitados, afirmamos as pesquisas feitas por Mourouzis e Koumoura, (2005) e Ruslin, et al., (2016), onde concluem que o futebol é o principal esporte causador de traumas faciais quando comparados a outros.

Um ponto a ser ressaltado é a falta da utilização dos protetores bucais e faciais, sendo apenas levantado este ponto nas pesquisas de Braham et al, (2004), Azodo, et al., (2011), Hwang e Kim, (2019). Medidas preventivas essenciais durante os jogos, já que estes, reduzem ou impedem significativamente, que as lesões maxilofaciais ocorram. Braham, et al, (2004), relata em seu estudo, que dos 301 jogadores entrevistados, 294 não usavam proteção facial ou bucal, ou seja, apenas 2,3\% dos jogadores em questão, usavam esse equipamento, enquanto $97,7 \%$ estavam sujeitos ao risco de fraturas graves e lesões não graves.

O tratamento dessas fraturas em suma maioria dos estudos, foi de forma conservadora. A pesquisa descrita por Antoun e Lee (2008), que utiliza em seu estudo a redução aberta e fixação interna, em que destaca que o uso da técnica de redução aberta obteve um número maior (60,3\%) em relação a outras técnicas aplicadas quando os jogadores se acidentavam.

\section{Conclusão}

Esta revisão revelou uma alta prevalência de lesões maxilofaciais, com prevalência maior na região da mandíbula e complexo zigomático. O esporte mais envolvido em traumas, foi o futebol, seguido do rugby. A forma de tratamento mais utilizada foi a conservadora. Apenas três autores, Braham, Azodo e Hwang enfatizaram o uso de protetores bucais, onde contribuíram significativamente na redução dessas lesões.

\section{Referências}

Antoun, J. S., \& Lee, K. H. (2008). Sports-related maxillofacial fractures over an 11-year period. J Oral Maxillofac Surg. 66(3):504-508.

Azodo, C., Odai, C., Osazuwa-Peters, N., \& Obuekwe, O. (2011). A survey of orofacial injuries among basketball players. International Dental Journal. 61: $43-46$.

Braham, R., Finch, C., \& McCrory, P. (2004) P. The incidence of head/neck/orofacial injuries in non-elite Australian Football. J Sci Med Sport. 7:4:451-453.

Carroll, S., Jawad, M., West, M., \& O’Connor, T. (1995). One hundred and ten sports related facial fractures. Br J Sport Med. 29: 194-195

Delilbasi, C., Yamazawa, M., Nomura, K., Iida, S., \& Kogo, M. (2004). Maxillofacial fractures sustained during sports played wiith a ball. Oral Surg Oral Med Oral Pathol Radiol Endod. 97: 23-2.

Frontera, R. Traumatismo orofacial durante a prática de basquetebol e nível de informação sobre trauma e protetor bucal. (2008). Dissertação (Mestrado em Odontologia). Universidade São Leopoldo Mandic. Campinas.

Hill, C., Burford, K., Martin, A., \& Thomas, D. (1998). A one-year review of maxillofacial sports injuries treated at an accident and emergency department. Brit J Oral Maxillofac Surg. 36:44-7.

Hwang, K., \& Kim, H. (2019). Facial Injuries in Handball: A Survey of Handball Coaches. Journal of Craniofacial Surgery. $30746-752$.

Lim, L., Moore, M., Trott, J., \& David, D. (1993). Sports related facial fractures: a review of 137 patients. Aust N Z J Surg. 63: $784-789$.

Maladiere, E., Bado, F., Meningaud, J., Guilbert, F., \& Bertrand, J. (2001). Aetiology and incidence of facial fractures sustained during sports: a prospective study of 140 patients. Int J Oral Maxillofac Surg.: 30: 291-295.

Motamedi, M. (2003). An assessment of maxillofacial fractures: a 5-year study of 237 patients. J Oral Maxillofac Surg 61: 61-64.

Mourouzis, C., \& Koumoura, F. (2005). Sports-related maxillofacial fractures: A retrospectivestudy of 125 patients. International Journal of Oral and Maxillofacial Surgery. 34(6), 635-638.

Murphy, C., O'Connell, J., Kearns, G., \& Stassen, L. (2015). Sports-Related Maxillofacial Injuries. J Craniofac Surg. 2015;26(7):2120-2123.

Ranalli, D., \& Demas, P. (2002). Orofacial injuries from sport: preventive measures for sports medicine. Sports Med.: 2:409-418. 
Research, Society and Development, v. 10, n. 16, e86101623378, 2021

(CC BY 4.0) | ISSN 2525-3409 | DOI: http://dx.doi.org/10.33448/rsd-v10i16.23378

Ruslin, M., Boffano, P., ten Brincke, Y., Forouzanfar, T., \& Brand, H. (2016). Sport-Related Maxillo-Facial Fractures. J Craniofac Surg. $27(1)$ :e91-e94.

Sane, J., \& Ylipaavalniemi, P. (1987). Maxillofacial and dental soccer injuries in Finland. British Journal of Oral and Maxillofacial Surgery. $25,383-390$.

Santos, A., Gimenez, L., Rebouças, C., Correa Filho, S., \& Rennó, T. (2004). Esportes no Brasil: situação atual e propostas para desenvolvimento. BNDES.

Tanaka, N., Hayashi, S., Amagasa, T., \& Kohama, G. (1996). Maxillofacial fractures sustained during sports. J Oral Maxillofac Surg.54:715-19.

Yamada, T. Sawaki, Y., Tomida, S., Tohnai, I., \& Ueda, M. (1998). Oral injury and mouthguard usage by athletes in Japan. Endod. Dent. Tramol. 14(2). 8487, 2008. 